\title{
Intuitionistic choice and restricted classical logic
}

\author{
Ulrich Kohlenbach \\ BRICS* \\ Department of Computer Science \\ University of Aarhus \\ Ny Munkegade \\ DK-8000 Aarhus C, Denmark \\ kohlenb@brics.dk
}

Keywords: Axiom of choice, intuitionistic arithmetic, restricted classical logic, König's lemma, primitive recursive arithmetic.

AMS Classification: 03F10, 03F35, 03F50, 03D15, 68Q15.

\begin{abstract}
Recently, Coquand and Palmgren considered systems of intuitionistic arithmetic in all finite types together with various forms of the axiom of choice and a numerical omniscience schema (NOS) which implies classical logic for arithmetical formulas. Feferman subsequently observed that the proof theoretic strength of such systems can be determined by functional interpretation based on a non-constructive $\mu$-operator and his well-known results on the strength of this operator from the 70's.

In this note we consider a weaker form LNOS (lesser numerical omniscience schema) of NOS which suffices to derive the strong form of binary König's lemma studied by Coquand/Palmgren and gives rise to a new and mathematically strong semi-classical system which, nevertheless, can proof theoretically be reduced to primitive recursive arithmetic PRA. The proof of this fact relies on functional interpretation and a majorization technique developed in a previous paper.
\end{abstract}

${ }^{*}$ Basic Research in Computer Science, Centre of the Danish National Research Foundation. 
In [6], systems of intuitionistic arithmetic in all finite types extended by various kinds of the axiom of choice and the schema of numerical omniscience

$$
\text { NOS: } \equiv \forall n(A(n) \vee \neg A(n)) \rightarrow \forall n A(n) \vee \exists n \neg A(n),
$$

where $n$ ranges over the natural numbers and $A$ is any formula ${ }^{1}$, are studied.

In [5], Feferman noticed that the proof theoretic strength of such systems can be determined by functional interpretation using his non-constructive $\mu$-operator and his classical results on the strength of systems based on this operator (see [1] for a survey of those results).

In this note we show that a similar use of functional interpretation combined with the majorization arguments which we developed in [8] can be used to determine the strength of systems which instead of NOS are based on the weaker schema of lesser numerical omniscience

$$
\text { LNOS }: \equiv\left\{\begin{array}{l}
\forall n^{0}((A(n) \vee \neg A(n)) \wedge(B(n) \vee \neg B(n))) \wedge \\
\neg(\exists n A(n) \wedge \exists n B(n)) \rightarrow \forall n \neg A(n) \vee \forall n \neg B(n),
\end{array}\right.
$$

which generalizes the well-known 'lesser limited principle of omniscience' (see [2] for various equivalent formulations of this principle)

$$
\text { LLOP }: \equiv \forall f^{1}, g^{1}(\neg(\exists n(f n=0) \wedge \exists n(g n=0)) \rightarrow \forall n(f n \neq 0) \vee \forall n(g n \neq 0))
$$

in the same way as NOS generalizes

$$
\mathbf{L P O}: \equiv \forall f^{1}(\forall n(f n=0) \vee \exists n(f n \neq 0)) .
$$

We will define a system based on LNOS and the full axiom schema of choice AC which allows to prove the version of König's lemma studied in [6] and is $\Pi_{2^{-}}^{0}$ conservative over PRA.

In the following $\mathbf{H A}^{\omega}$ and $\widehat{\mathbf{H A}}^{\omega}$ are the systems of arithmetic in all finite types denoted by WE-HA ${ }^{\omega}$ and WE- $\widehat{\mathrm{HA}}^{\omega}$ in [1], where, however, the quantifier-free rule of extensionality is defined as

$$
\frac{\vdash A_{0} \rightarrow s={ }_{\rho} t}{\vdash A_{0} \rightarrow r[s]={ }_{\tau} r[t]}
$$

\footnotetext{
${ }^{1} A$ may contain arbitrary parameters.
} 
where $A_{0}$ is quantifier-free. ${ }^{2} \widehat{\mathbf{H A}}^{\omega}$ contains only recursion on type 0 and induction restricted to $\Sigma_{1}^{0}$-formulas. $\widehat{\mathbf{H A}}^{\omega} \wedge$ is the still weaker system with quantifier-free induction only.

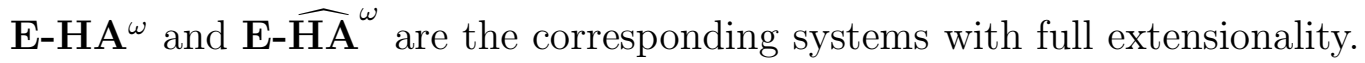

The axiom schema of choice is given by

$$
\mathbf{A C}^{\rho, \tau}: \forall x^{\rho} \exists y^{\tau} A(x, y) \rightarrow \exists Y^{\rho \rightarrow \tau} \forall x^{\rho} A(x, Y x), \quad \mathbf{A C}:=\bigcup_{\rho, \tau}\left\{\mathbf{A C}^{\rho, \tau}\right\}
$$

The axiom schema of unique choice is given by

$$
\mathbf{A C} !^{\rho, \tau}: \forall x^{\rho} \exists ! y^{\tau} A(x, y) \rightarrow \exists Y^{\rho \rightarrow \tau} \forall x^{\rho} A(x, Y x) .
$$

Lemma $1 \mathbf{H A}^{\omega}+\mathbf{A C} \mathbf{C}^{0,0}+\mathbf{L L O P} \vdash \mathbf{L N O S . ~ S i m i l a r l y ~ f o r ~} \widehat{\mathbf{H A}}^{\omega} \wedge$ instead of $\mathbf{H A}^{\omega}$.

Proof: By intuitionistic logic and $0 \neq 1$ one proves that

$$
\forall n^{0}(A(n) \vee \neg A(n)) \rightarrow \forall n^{0} \exists k^{0}([k=0 \rightarrow A(n)] \wedge[k \neq 0 \rightarrow \neg A(n)]) .
$$

By $\mathbf{A C}^{0,0}$ and the stability of $=_{0}$ this yields

$$
\exists f \forall n(f(n)=0 \leftrightarrow A(n)) .
$$

Likewise, we get a characteristic function for $B(n)$. So by applying LLOP to $f, g$ we obtain LNOS.

In the following, $\mathbf{M}^{\omega}$, $\mathbf{I P}_{0}^{\omega}$ denote the Markov principle resp. the 'independence-ofpremise principle' from [11](3.5.10).

Theorem 2 1) $\mathbf{H A}^{\omega}+\mathbf{A C}+\mathbf{M}^{\omega}+\mathbf{I P}_{0}^{\omega}+\mathbf{L N O S}$ is $\Pi_{2}^{0}$-conservative over $\mathbf{H A}$.

2) $\widehat{\mathbf{H A}}^{\omega}+\mathbf{A C}+\mathbf{M}^{\omega}+\mathbf{I P}_{0}^{\omega}+\mathbf{L N O S}$ is $\Pi_{2}^{0}$-conservative over PRA.

If $\mathbf{A C}$ is replaced by $\mathbf{A C}^{0, \tau}$ plus $\mathbf{A C} !^{1, \tau}$ (with arbitrary $\tau$ ) and $\mathbf{M}^{\omega}$ and $\mathbf{I P}_{0}^{\omega}$ are restricted to instances containing only quantified variables of types $\leq 1$, then the above conservation results also hold for the fully extensional systems $\mathbf{E}-\mathbf{H A}^{\omega}$ and E- $\widehat{\mathbf{H A}}^{\omega}$.

\footnotetext{
2 ' $\vdash$ ' indicates that further non-logical axioms are not allowed to be used in the proof of a premise of that rule. This restriction is necessary for the deduction theorem to hold true which we will use below. Alternatively, one could (as we have done in previous papers) formulate the rule without this restriction and then using instead a special symbol $\oplus$ to indicate that in WE-HA ${ }^{\omega} \oplus A$ the axiom $A$ is not allowed to be used in any proof of the premise of QF-ER.
} 
Proof: 1) By the lemma above it is sufficient to consider LLOP. So let

$$
\mathbf{H A}^{\omega}+\mathbf{A C}+\mathbf{M}^{\omega}+\mathbf{I P}_{0}^{\omega} \vdash \mathbf{L L O P} \rightarrow \forall x \exists y R(x, y),
$$

where $\forall x \exists y R(x, y)$ is a $\Pi_{2}^{0}$-sentence in $\mathcal{L}(\mathbf{H A})$.

Relative to $\mathbf{H A}^{\omega}$ we can write LLOP equivalently as

$$
\forall n, \tilde{n}(f n \neq 0 \vee g \tilde{n} \neq 0) \rightarrow \exists k \leq 1([k=0 \rightarrow \forall n(f n \neq 0)] \wedge[k \neq 0 \rightarrow \forall n(g n \neq 0)]) .
$$

The latter is implied by

$$
\exists k \leq 1 \forall z(\underbrace{\forall n, \tilde{n} \leq z(f n \neq 0 \vee g \tilde{n} \neq 0) \rightarrow([k=0 \rightarrow f z \neq 0] \wedge[k \neq 0 \rightarrow g z \neq 0])}_{A_{0}(f, g, k, z): \equiv}),
$$

where $A_{0}$ can be written as a quantifier-free formula. Hence

$$
(*) \mathbf{H A}^{\omega}+\mathbf{A C}+\mathbf{M}^{\omega}+\mathbf{I P}_{0}^{\omega} \vdash \forall f, g \exists k \leq 1 \forall z A_{0}(f, g, k, z) \rightarrow \forall x \exists y R(x, y) .
$$

By a combination of functional interpretation and majorization as used in [8] one can reduce the use of

$$
\forall f, g \exists k \leq 1 \forall z A_{0}(f, g, k, z)
$$

to

$$
\forall f, g, z \exists k \leq 1 \forall \tilde{z} \leq z A_{0}(f, g, k, \tilde{z}) .
$$

For the sake of completeness we sketch the proof here: $(*)$ implies

$$
\mathbf{H A}^{\omega}+\mathbf{A C}+\mathbf{M}^{\omega}+\mathbf{I P}_{0}^{\omega} \vdash \forall x \forall F \leq{ }_{\rho} 1^{\rho} \exists f, g, z, y\left(A_{0}(f, g, F f g, z) \rightarrow R(x, y)\right),
$$

where $\rho:=1 \rightarrow(1 \rightarrow 0), \leq_{\rho}$ is defined pointwise and $1^{\rho}:=\lambda f, g .1$.

By functional interpretation (see [11](3.5.10)) one extracts a closed term $\Phi$ of $\mathbf{H A}^{\omega}$ such that

$$
\mathbf{H A}^{\omega} \vdash \forall x \forall F \leq 1\left(\forall f, g A_{0}(f, g, F f g, \Phi F x) \rightarrow \exists y R(x, y)\right) .
$$

By [7], $\Phi$ has a majorizing functional $\Phi^{*}$ and hence (using basic properties of majorization in Howard's sense)

$$
\mathbf{H A}^{\omega} \vdash \forall x \forall F \leq 1\left(t x:=\Phi^{*} 1^{\rho} x \geq \Phi F x\right) .
$$

Put together we get

$$
\mathbf{H A}^{\omega} \vdash \forall x \forall F \leq 1\left(\forall f, g \forall z \leq t x A_{0}(f, g, F f g, z) \rightarrow \exists y R(x, y)\right)
$$


and hence

$$
\mathbf{H A}^{\omega} \vdash \forall z \exists F \leq 1 \forall f, g \forall \tilde{z} \leq z A_{0}(f, g, F f g, \tilde{z}) \rightarrow \forall x \exists y R(x, y) \text {. }
$$

Since $F$ can be obtained by primitive recursive definition by cases this yields

$$
\mathbf{H A}^{\omega} \vdash \forall f, g, z \exists k \leq 1 \forall \tilde{z} \leq z A_{0}(f, g, k, \tilde{z}) \rightarrow \forall x \exists y R(x, y) .
$$

However, $\forall f, g, z \exists k \leq 1 \forall \tilde{z} \leq z A_{0}(f, g, k, \tilde{z})$ can easily be verified in $\mathbf{P A}^{\omega}$ and hence (using negative translation and the fact that this statement can be written as a purely universal sentence) in $\mathbf{H A}^{\omega}$. Thus $\mathbf{H A}^{\omega} \vdash \forall x \exists y R(x, y)$. The theorem now follows by the well-known conservation of $\mathbf{H A}^{\omega}$ over $\mathbf{H A}$.

2) The proof is analogous to 1) using that $\widehat{\mathbf{P A}}^{\omega}$ has a negative translation into $\widehat{\mathbf{H A}}^{\omega}+\mathbf{M}^{\omega}$ and the latter has a functional interpretation in $\widehat{\mathbf{H A}}^{\omega} \uparrow$ which is $\Pi_{2}^{0}-$ conservative over PRA.

The claim for the fully extensional systems follows by the well-known elimination of extensionality technique (see [10] for details).

[6] introduces an extension of the usual weak König's lemma WKL to binary trees given by arbitrary formulas $\Phi(\underline{x}, m)$ which are decidable in the variable $m$ which defines the tree, i.e. $\forall m(\Phi(\underline{x}, m) \vee \neg \Phi(\underline{x}, m))$. Let's call that schema DWKL (see [6] p.57 for details).

Theorem 3 Both $\mathbf{H A}^{\omega}+\mathbf{A} \mathbf{C}^{0,0}+\mathbf{L N O S}$ and $\widehat{\mathbf{H A}}^{\omega} \uparrow+\mathbf{A C}^{0,0}+\mathbf{L N O S}$ prove DWKL.

Proof: We show the theorem for $\widehat{\mathbf{H A}}^{\omega} \uparrow+\mathbf{A C}^{0,0}+\mathbf{L N O S}$. Analogously to the proof of the lemma above one verifies that $\widehat{\mathbf{H A}}^{\omega} \Lambda+\mathbf{A} \mathbf{C}^{0,0}$ allows us to reduce DWKL to the usual weak König's lemma WKL as defined in [12]:

$\mathrm{WKL}: \equiv \forall f^{1}\left(T(f) \wedge \forall x^{0} \exists n^{0}(\operatorname{lth}(n)=x \wedge f n=0) \rightarrow \exists b^{1} \forall x^{0}(f(\bar{b} x)=0)\right)$, where $T f: \equiv \forall n^{0}, m^{0}\left(f(n * m)={ }_{0} 0 \rightarrow f n={ }_{0} 0\right) \wedge \forall n^{0}, x^{0}\left(f(n *\langle x\rangle)={ }_{0} 0 \rightarrow x \leq_{0} 1\right)$.

Consider the formula ${ }^{3}$

$$
(+)\left\{\begin{array}{l}
\forall x^{0} \exists n \leq_{0} 1 \forall k>0(\exists m \leq \overline{1} k(l t h(m)=k \wedge f(x * m)=0) \\
\rightarrow \exists m \leq \overline{1}(k-1)(l \operatorname{th}(m)=k-1 \wedge f(x *\langle n\rangle * m)=0)) .
\end{array}\right.
$$

\footnotetext{
${ }^{3}$ Here we use that our coding of finite sequences has the property that $\forall n, m, f, g(n \geq m \wedge \forall x(f x \geq g x) \rightarrow \bar{f} n \geq \bar{g} m)$, which can be arranged.
} 
We first show that $\widehat{\mathbf{P A}}^{\omega} \wedge \vdash T(f) \rightarrow(+)$, where $\widehat{\mathbf{P A}}^{\omega} \wedge$ is the classical counterpart of $\widehat{\mathrm{HA}}^{\omega} \wedge$ : Let $x$ be arbitrary but fixed.

Case 1: $\forall k>0 \exists m \leq \overline{1} k(l t h(m)=k \wedge f(x * m)=0)$.

Then classical logic yields (using $T(f)$ )

$$
\begin{aligned}
& \forall k>0 \exists m \leq \overline{1} k(l t h(m)=k \wedge f(x *\langle 0\rangle * m)=0) \vee \\
& \forall k>0 \exists m \leq \overline{1} k(l t h(m)=k \wedge f(x *\langle 1\rangle * m)=0) .
\end{aligned}
$$

Set $n=0$ in the case where the first disjunct is true, and set $n=1$ otherwise.

Case 2: $\exists k>0 \neg \exists m \leq \overline{1} k(l t h(m)=k \wedge f(x * m)=0)$. By the quantifier-free leastnumber-principle (hence by the schema QF-IA of quantifier-free induction) we find the least such $k$. Call it $k_{0}$.

2.1: $k_{0}=1$ : Choose $n \leq 1$ arbitrarily.

2.2: $k_{0}>1:$ Then

$$
\exists m \leq \overline{1}\left(k_{0}-1\right)\left(l t h(m)=k_{0}-1 \wedge f(x * m)=0\right) .
$$

choose $n:=(m)_{0}$ for such an $m$. This finishes the proof of $\widehat{\mathbf{P A}}^{\omega} \wedge \vdash T(f) \rightarrow(+)$. By negative translation we get

$$
\widehat{\mathbf{H A}}^{\omega} \wedge \vdash T(f) \rightarrow(+)^{\prime}
$$

where

$$
(+)^{\prime}: \equiv\left\{\begin{array}{l}
\forall x^{0} \neg \neg \exists n \leq_{0} 1 \forall k>0(\exists m \leq \overline{1} k(l t h(m)=k \wedge f(x * m)=0) \\
\rightarrow \exists m \leq \overline{1}(k-1)(l \operatorname{th}(m)=k-1 \wedge f(x *\langle n\rangle * m)=0)) .
\end{array}\right.
$$

But $\widehat{\mathbf{H A}}^{\omega} \uparrow+\mathbf{L L O P} \vdash(+)^{\prime} \rightarrow(+)$. Hence

$$
\widehat{\mathbf{H A}}^{\omega} \uparrow+\mathbf{L L O P} \vdash T(f) \rightarrow(+) .
$$

Assume $T(f) \wedge \forall x \exists n(l \operatorname{th}(n)=x \wedge f n=0)$.

By applying $\mathbf{A} \mathbf{C}^{0,0}$ to $(+)$ we get a function $g$ such that

$$
\left\{\begin{array}{l}
\forall x^{0}\left(g x \leq_{0} 1 \wedge \forall k>0(\exists m \leq \overline{1} k(l t h(m)=k \wedge f(x * m)=0)\right. \\
\rightarrow \exists m \leq \overline{1}(k-1)(l \operatorname{th}(m)=k-1 \wedge f(x *\langle g x\rangle * m)=0))) .
\end{array}\right.
$$


Define $\tilde{h}(0):=\langle\rangle, \tilde{h}(n+1):=\tilde{h}(n) *\langle g(\tilde{h}(n))\rangle$.

Now take $h(n):=(\tilde{h}(n+1))_{n}$. By quantifier-free induction we show that $(++) \forall n(\tilde{h}(n)=\bar{h}(n))$ :

$n=0: \quad \tilde{h}(0)=\langle\rangle=\bar{h}(0)$.

$n \rightarrow n+1: \tilde{h}(n+1)=\tilde{h}(n) *\langle g(\tilde{h} n)\rangle \stackrel{\text { I.H. }}{=} \bar{h} n *\langle g(\tilde{h} n)\rangle \stackrel{\operatorname{lth}(\tilde{h} n)=n}{=} \bar{h}(n) *\left\langle(\tilde{h}(n+1))_{n}\right\rangle=$ $\bar{h}(n) *\langle h n\rangle=\bar{h}(n+1)$.

Let $k$ be arbitrary but fixed. We now show - again by quantifier-free induction on $n$ - that

$$
\forall n<k \exists m \leq \overline{1}(k-n)(l t h(m)=k \cdot n \wedge f(\bar{h}(n) * m)=0):
$$

$n=0: \bar{h}(0) * m=m$, hence the claim follows from $T(f) \wedge \forall x \exists n(l t h(n)=x \wedge f n=0)$. $n \rightarrow n+1$ : We may assume that $n+1<k$ : By I.H.

$$
\exists \tilde{m} \leq \overline{1}(k-n)(l t h(\tilde{m})=k-n \wedge f(\bar{h}(n) * \tilde{m})=0) .
$$

Hence by $g$-definition

$$
\exists m \leq \overline{1}(k-(n+1))(l t h(m)=k \bullet(n+1) \wedge f(\underbrace{\bar{h} n *\langle g(\bar{h} n)\rangle}_{=\bar{h}(n+1)(++)} * m)=0),
$$

which is the claim for $n+1$.

So in total we have shown that $T(f) \wedge \forall x \exists n(l t h(n)=x \wedge f n=0)$ implies

$$
\forall k \forall n<k \exists m \leq \overline{1}(k-n)(l t h(m)=k \cdot n \wedge f(\bar{h}(n) * m)=0)
$$

and hence

$$
\forall n(f(\bar{h} n)=0)
$$

i.e. $h$ satisfies WKL.

Corollary to the proof of the theorem: In the proof of the theorem above we have only used elementary recursive functionals from $\widehat{\mathbf{H A}}^{\omega} \uparrow$. So the argument also applies to even weaker systems having the strength of Kalmar elementary arithmetic EA.

Remark 4 By combining theorems 2 and 3 proved above, one concludes that the strong version of (weak) König's lemma from [6] DWKL may be added to the systems in question without destroying the conservation results. Instead of the rather tedious proof of weak König's lemma from LLOP and $\mathbf{A C}^{0,0}$ one could also more easily directly apply the proof of theorem 2 to the situation where weak König's lemma is added and use the WKL-elimination from [8]. However, we preferred the first route as an application of LLOP. 
Remark 5 If one is not interested in proof theoretic reductions to systems of low proof theoretic strength but in the more applied aspect of extracting algorithms or bounds from proofs of semi-classical systems, then (at least in the absence of $\left.\mathbf{M}^{\omega}\right)^{4}$ much stronger results can be obtained as we have shown in [9]. E.g. consider the comprehension principle for negated formulas in all types

$$
\mathbf{C A}_{\neg}: \exists \Phi^{\rho \rightarrow 0} \forall x^{\rho}(\Phi(x)=0 \leftrightarrow \neg A(x))
$$

(where $A$ is an arbitrary formula) and the full double negation shift schema

$$
\text { DNS : } \forall x^{\rho} \neg \neg A \rightarrow \neg \neg \forall x^{\rho} A
$$

and define $\mathcal{T}:=\widehat{\mathbf{H A}}^{\omega}+\mathbf{A C}+\mathbf{D N S}+\mathbf{C A}_{\neg}$. Then the provable functions of $\mathcal{T}$ are bounded by primitive recursive functions although $\mathcal{T}$ allows us to interpret full classical type theory via negative translation. For weak subsystems instead of $\widehat{\mathbf{H A}}^{\omega}$, even polynomial bounds are guaranteed.

Remark 6 Intuitionistically one can allow certain induction principles which classically would go beyond the strength of PRA and still obtain conservation over PRA. E.g. [13] considers function parameter free forms of induction rules for fomulas like $\exists f^{1} \forall x^{0} A_{0}$ (with quantifier-free $A_{0}$ ). It seems likely that also in this context one may add LNOS and still preserve PRA-reducibility.

\section{References}

[1] Avigad, J., Feferman, S., Gödel's functional ('Dialectica') interpretation. In: [3], pp. 337-405 (1998).

[2] Bridges, D., Richman, F., Varieties of Constructive Mathematics. London Mathematical Society LNS 97, Cambridge University Press, pp. x+149 (1987).

[3] Buss, S.R. (editor), Handbook of Proof Theory. Studies in Logic and the Foundations of Mathematics Vol 137, Elsevier, vii+811 pp. (1998).

[4] Feferman, S., Theories of finite type related to mathematical practice. In: Barwise, J. (ed.), Handbook of Mathematical Logic, pp. 913-972, North-Holland, Amsterdam (1977).

\footnotetext{
${ }^{4}$ For a strong result in this direction in the presence of $\mathbf{M}^{\omega}$ see [9](thm.3.18).

${ }^{5}$ Not only provably recursive functions!
} 
[5] Feferman, S., On the proof theoretical strength of some systems with the numerical omniscience scheme (abstract, ASL Summer Meeting, Paris 2000). To appear in Bull. Symbolic Logic.

[6] Coquand, T., Palmgren, E., Intuitionistic choice and classical logic. Arch. Math. Logic 39, pp. 53-74 (2000).

[7] Howard, W.A., Hereditarily majorizable functionals of finite type. In [11], pp. 454-461.

[8] Kohlenbach, U., Effective bounds from ineffective proofs in analysis: an application of functional interpretation and majorization. J. Symbolic Logic 57, pp. 1239-1273 (1992).

[9] Kohlenbach, U., Relative constructivity. J. Symbolic Logic 63, pp. 1218-1238 (1998).

[10] Luckhardt, H., Extensional Gödel functional interpretation. A consistency proof of classical analysis. Springer Lecture Notes in Mathematics 306 (1973).

[11] Troelstra, A.S. (ed.) Metamathematical investigation of intuitionistic arithmetic and analysis. Springer Lecture Notes in Mathematics 344 (1973).

[12] Troelstra, A.S., Note on the fan theorem. J. Symbolic Logic 39, pp. 584-596 (1974).

[13] Ye, F., Strict Constructivism and the Philosophy of Mathematics. Ph.D. Thesis, Princeton University 1999. 